\title{
Spinal Efference Copy Signaling and Gaze Stabilization during Locomotion in Juvenile Xenopus Frogs
}

\author{
Géraldine von Uckermann, ${ }^{1}$ Didier Le Ray, ${ }^{1}$ Denis Combes, ${ }^{1}$ Hans Straka, ${ }^{2 *}$ and John Simmers ${ }^{1 *}$ \\ ${ }^{1}$ Université Bordeaux, Institut de Neurosciences Cognitives et Intégratives d'Aquitaine, Centre National de la Recherche Scientifique Unité Mixte de \\ Recherche 5287, 33076 Bordeaux, France, and ${ }^{2}$ Centre d'Etudes de la SensoriMotricité, Centre National de la Recherche Scientifique Unité Mixte de \\ Recherche 8194, Université Paris Descartes, 75006 Paris, France
}

In swimming Xenopus laevis tadpoles, gaze stabilization is achieved by efference copies of spinal locomotory CPG output that produce rhythmic extraocular motor activity appropriate for minimizing motion-derived visual disturbances. During metamorphosis, Xenopus switches its locomotory mechanism from larval tail-based undulatory movements to bilaterally synchronous hindlimb kick propulsion in the adult. The change in locomotory mode leads to body motion dynamics that no longer require conjugate left-right eye rotations for effective retinal image stabilization. Using in vivo kinematic analyses, in vitro electrophysiological recordings and specific CNS lesions, we have investigated spino-extraocular motor coupling in the juvenile frog and the underlying neural pathways to understand how gaze control processes are altered in accordance with the animal's change in body plan and locomotor strategy. Recordings of extraocular and limb motor nerves during spontaneous "fictive" swimming in isolated CNS preparations revealed that there is indeed a corresponding change in spinal efference copy control of extraocular motor output. In contrast to fictive larval swimming where alternating bursts occur in bilateral antagonistic horizontal extraocular nerves, during adult fictive limb-kicking, these motor nerves are synchronously active in accordance with the production of convergent eye movements during the linear head accelerations resulting from forward propulsion. Correspondingly, the neural pathways mediating spino-extraocular coupling have switched from contralateral to strictly ipsilateral ascending influences that ensure a coactivation of bilateral extraocular motoneurons with synchronous left-right limb extensions. Thus, adaptive developmental plasticity during metamorphosis enables spinal CPG-driven extraocular motor activity to match the changing requirements for eye movement control during self-motion.

\section{Introduction}

Vertebrate locomotion and the resultant body and head displacements require offsetting eye movements to ensure continuous accurate perception of the visual world. Such gaze-stabilizing adjustments, in correspondence with the specific locomotor strategy used, are traditionally attributed to the transformation of movement-detecting sensory inputs into appropriate extraocular motor commands (Angelaki and Hess, 2005). Accordingly, visuo-vestibular and proprioceptive reflexes generate eye movements that counteract body/head motion, thereby minimizing retinal image drift (Straka and Dieringer, 2004). Recently, however, an alternative gaze-stabilizing mechanism has been re-

Received Sept. 23, 2012; revised Nov. 27, 2012; accepted Dec. 23, 2012.

Author contributions: D.C., H.S., and J.S. designed research; G.v.U., D.L.R., and D.C. performed research; G.v.U. and D.L.R. analyzed data; G.v.U., H.S., and J.S. wrote the paper.

This work was supported by Agence Nationale de la Recherche (ANR-08-BLAN-0145-01, Galodude), the German Science Foundation (CRC 870, B12), the German Federal Ministry of Education and Research (01 E0 0901), and Bayerisch-Französisches Hochschulzentrum.

The authors declare no competing financial interests.

*H.S. and J.S. contributed equally to this work.

Correspondence should be addressed to Dr. John Simmers, Université Bordeaux Segalen, Centre National de la

Recherche Scientifique Unité Mixte de Recherche 5287, Institut de Neurosciences Cognitives et Intégratives d'Aquitaine, 146 rue Léo Saignat, 33076 Bordeaux, France. E-mail: john.simmers@u-bordeaux2.fr.

H. Straka's present address is Biocenter-Martinsried, Department II, Faculty of Biology, Ludwig-MaximiliansUniversität Munich, Grosshadernerstr. 2, 82152 Planegg, Germany.

DOI:10.1523/JNEUROSCI.4521-12.2013

Copyright $\odot 2013$ the authors $\quad 0270-6474 / 13 / 334253-12 \$ 15.00 / 0$ ported in swimming larval Xenopus, whereby classical reflexdriven eye movements were found to be supplanted by an intrinsic predictive mechanism that uses efference copy signals arising from the locomotor CPG itself (Lambert et al., 2012), and thus with potentially novel implications for vertebrate gaze control in general.

In the biomechanically compact tadpole, the use of spinal feedforward signals as a mechanism for driving corrective eye movements may be related to its relatively simple tail-based swimming behavior during which predictable left-right head rotations occur in the horizontal plane (Hoff and Wassersug, 1986; Roberts et al., 1998; Azizi et al., 2007). However, because maintaining visual acuity during self-motion is a requirement for aquatic and terrestrial vertebrates alike, the question arises as to whether spinal CPG circuitry also contributes to gaze stabilization in animals confronted with more complex visual disturbances arising from flexible necks and/or limb-based modes of locomotion (Chagnaud et al., 2012). In this respect, the Xenopus frog, with its employment of fundamentally different propulsive mechanisms before and after metamorphosis, offers a unique opportunity to study within a single species the adaptive plasticity in gaze control and the role of spinal efference copy signaling in an organism that lies at the interface between two principal locomotor strategies in the animal kingdom. In contrast to tadpole axial swimming, postmetamorphic froglets produce body displacements by rhythmic, bilaterally synchronous hindlimb 
kicking (Combes et al., 2004; Beyeler et al., 2008), with an accompanying switch from horizontal angular head rotations (Lambert et al., 2012) to linear forward acceleration and then deceleration in each locomotor cycle (Straka and Dieringer, 2004). Consequently, instead of conjugate left-right eye oscillations during larval locomotion, the gaze-stabilizing process in the swimming adult is substantially different, now requiring binocular movements appropriate for offsetting the disruptive visual effects of longitudinal body/head motion (Rohregger and Dieringer, 2002).

Here, kinematic analyses of eye motion in deafferented juvenile frogs during swimming revealed that convergent eye movements occur during the rectilinear propulsive phase of each locomotor cycle. Compatible with this behavioral coordination, locomotor activity expressed spontaneously in isolated CNS preparations, and therefore devoid of sensory feedback, consisted of rhythmic in-phase burst discharge in bilateral hindlimb extensor nerves and medial rectus extraocular motoneurons. This spino-extraocular coordination in vitro, together with the effects of specific CNS lesions, indicated that the coupling is mediated by direct, homolateral pathways arising from the lumbar cord region. Thus, locomotor efference copies do indeed contribute to gaze stabilization in a tetrapod in accordance with the particular spatiotemporal characteristics of its propulsive strategy and associated visual demands.

\section{Materials and Methods}

Animals. Experiments were performed on the South African clawed toad Xenopus laevis $(n=51)$ of either sex at developmental stages 64-66 (Nieuwkoop and Faber, 1956), bred from an in-house laboratory colony or obtained from an authorized supplier and maintained at $20-22^{\circ} \mathrm{C}$ in aquaria exposed to a $12 \mathrm{~h}: 12 \mathrm{~h}$ light/dark cycle. The animals used in this study had snout-vent lengths of $1.3-1.9 \mathrm{~cm}$ and weighed $\sim 1.25 \mathrm{~g}$. All experimental procedures were conducted in accordance with the European Communities Council Directive and the local ethics committee of the University of Bordeaux (\#3301100012-A).

Video analysis of limb kinematics and eye movement. To study hindlimb kinematics during free forward swimming, animals $(n=6)$ were placed in a Plexiglas aquarium $(8 \times 15 \mathrm{~cm})$ that was sufficiently large for the expression of up to 6 successive hindlimb-kick cycles and filled with water to a depth of $\sim 2-3 \mathrm{~cm}$ to ensure unobstructed swimming movements in the horizontal plane. In these experiments, only uninterrupted episodes of swimming with at least three consecutive kicks were analyzed. High-speed video analyses of eye movements were conducted on headrestrained semi-intact preparations $(n=3)$ placed in a Sylgard-lined Petri dish after severing both optic nerves and the removal of their vestibular endorgans by the ablation of the otic capsules (see below for details). The head of such preparations was firmly secured with insect pins while the hindlimbs were left free to perform swimming movements that were triggered by gently tapping the aquarium wall or by directly touching a leg. A ruler placed beneath the aquarium or on the side of the Petri dish was used for scaling the movements in video sequences recorded with a high-speed digital camera (Grasshopper 03K2C, Point Grey Research) positioned above the animal. A wide-angle varifocal lens (YV10x5B-2, Fujinon Europe) was used to monitor movement trajectories throughout free-swimming episodes, whereas a micro-inspection zoom lens system (Optem Zoom 70XL, Qioptiq Photonics) was used to record the eye motion during hindlimb swimming movements. Image acquisition frequencies were 60-200 fps for filming of limb movements and 115-225 fps for eye movement recordings. Video sequences were collected on computer through a FireWire interface and stored using the AVI video file format with software FlyCapture 1.8 (Point Grey Research). Subsequent data analyses were performed using free ImageJ $1.44 \mathrm{p}$ software (National Institute of Health) and a plug-in that enabled manual tracking of eye position throughout a recorded sequence of swimming activity and extracting the $x-y$ coordinates of predesignated marker points in relation to the corresponding hindlimb position. Based on these coordinates and the time interval between successive images, velocity and acceleration values were then calculated. All values are indicated as mean \pm SD. Regression analyses and plots were made using Origin 6.1 software (Microcal). For the purpose of illustration, individual images were extracted from video sequences using VirtualDub 1.9.11 free software.

Reduced preparation procedures. For experiments conducted both on semi-intact, hindlimb-attached preparations (for kinematic analysis) or completely isolated brainstem/spinal cord preparations (for electrophysiology), animals were first anesthetized under ice-chilled frog Ringer (composition in mM: NaCl, 93.54; KCl, 3; $\mathrm{NaHCO}_{3}, 30 ; \mathrm{CaCl}_{2}, 2.65$; $\left.\mathrm{MgCl}_{2}, 1 ; \mathrm{C}_{6} \mathrm{H}_{12} \mathrm{O}_{6}, 10.99 ; \mathrm{NaH}_{2} \mathrm{PO}_{4}, 0.5 ; \mathrm{pH} 7.4\right)$ containing $0.1 \%$ 3-aminobenzoic acid ethyl ester (MS-222; Sigma-Aldrich) and then secured with insect pins in a Sylgard-lined Petri dish after decerebration. The skull was opened and the telencephalon removed. For semi-intact preparations, both optic nerves were transected to omit visual inputs, and the bilateral vestibular endorgans were removed to exclude potential sensory inputs arising from any undetected head movements. After exposure of the rostral spinal cord for more effective oxygenation, preparations were rinsed in fresh saline and transferred to a second Sylgardlined Petri dish in which the floor was partly removed to allow unimpeded movements of the intact hindlimbs. Oxygenated Ringer's solution $\left(95 \% \mathrm{O}_{2}, 5 \% \mathrm{CO}_{2}\right)$ was supplied to the bath at a rate of $1.3-2.1$ $\mathrm{ml} / \mathrm{min}$ and maintained at $18 \pm 0.1^{\circ} \mathrm{C}$ with a Peltier cooling system.

For completely isolated brainstem/spinal cord preparations $(n=42)$, the motor nerve branches innervating the hindlimb tibialis anterior (ankle flexor, Flex) and plantaris longus (ankle extensor, Ext) muscles, respectively (d'Avella et al., 2003; d'Avella and Bizzi, 2005), were identified by dissecting distally until their individual target muscle and the spinal cord with the limb nerves attached was carefully removed from the rest of the trunk. The motor nerve branches innervating the medial and lateral rectus extraocular muscles on both sides were then dissected free and severed close to their respective muscles for recording impulse activity from the distal nerve stumps (see below). Thereafter, the preparation, including the brainstem and attached spinal cord, was rinsed in fresh saline, transferred to a recording chamber and secured ventral side up (Combes et al., 2004; Beyeler et al., 2008).

Electrophysiology. Simultaneous extracellular recordings (A-M Systems model $1700 \mathrm{AC}$ amplifiers) were made from the bilateral extraocular motor nerves, the hindlimb motor branches (Ext, Flex), and occasionally the ventral roots of the second thoracic cord segment (Th2) during episodes of spontaneous fictive swimming. Activity in limb Ext and Flex nerves was recorded with custom-made stainless steel wire electrodes isolated electrically with Vaseline, whereas the extraocular nerves and Th2 ventral roots were recorded with manipulator-positioned glass suction electrodes. Nerve activity was digitized at $10 \mathrm{kHz}$ (CED 1401, Cambridge Electronic Design) and displayed and stored on computer for offline analysis with Spike2 (CED) using customized scripts.

Brainstem/spinal cord lesions. In separate series of experiments, selective lesions of isolated preparations were made to disrupt neuronal communication within and between brainstem or spinal cord regions to determine the trajectory of the axonal pathways that mediate spinoextraocular coupling. Specific lesions in various combinations consisted of the following: (1) a sagittal midline separation of the cervicothoracic cord from segment C1 until Th3 ( $n=4$ animals); (2) a short midline sagittal incision at rhombomere 5 in the medial reticular formation $(n=$ 4); (3) a rostral cord hemi-section at the level of the obex $(n=2)$; (4) a longitudinal splitting that completely separated the two sides of the midbrain (diencephalon and mesencephalon; $n=3$ ) or of the entire brainstem until the obex (diencephalon, mesencephalon, and rhombencephalon; $n=2$ ); (5) an extensive longitudinal splitting that completely separated the two sides of the entire brainstem and the spinal cord until Th3 $(n=2)$; or $(6)$ a surgical ablation of the cerebellum $(n=2)$. All lesions were made with a razor blade fragment or with micro-scissors (Superfine Vannas Scissors, World Precision Instruments) depending on the extent of the lesion. In the case of the restricted lesion to rhombomere 5 , in which the abducens nucleus is located (Straka et al., 2001) and wherein abducens internuclear neurons traverse the brainstem midline 
to ascend in the medial longitudinal fascicle toward the oculomotor nucleus (Straka and Dieringer, 1991), the incision was guided using external anatomical landmarks. In particular, these included the VIIIth and IXth cranial nerves, which have been shown to delineate, respectively, the rostral and caudal borders of rhombomere 5 (Straka et al., 2006). The cerebellum was removed by cutting the cerebellar peduncle bilaterally at the brainstem insertion site. For all lesion experiments, a control period of at least $30 \mathrm{~min}$ was respected before electrophysiological data were collected, and the location and extent of lesions were verified by microscopic inspection after each experiment.

Electrophysiological data analysis and statistics. For single-experiment analyses, event correlations were generated from the raw electrophysiological recordings by setting event detection thresholds just above resting activity before the onset of an episode of fictive locomotion during which all suprathreshold activity was transferred into the corresponding Spike2 event channel. Event correlations were used to evaluate the phase relationship between the activities of selected nerve pairs during fictive swimming, with synchronous activity corresponding to a central peak in the corresponding cross-correlogram, whereas recurrent rhythmic bursting was indicated by regular satellite peaks on either side of the maximum. Correlation coefficients indicating the strength of coupling between the activities in nerve pairs were calculated in Matlab version 7.1 (Mathworks).

For multiple-experiment comparisons, the temporal relationships between discharges in the same nerve pairs in different preparations were assessed by circular phase analysis of pooled data using Oriana 2 software (Kovach Computing Services). The mean vector $\mu$ and its length $r$ indicated the preferred phase and the strength of coupling, respectively. For nonuniform distributions (tested with the Rayleigh's uniformity test, $Z$ ), phase values were plotted as the grand mean of the individual means of relative burst onsets throughout individual locomotor episodes. The effects of specific lesions on phase relationships were evaluated by using the Watson-Williams $F$ test to determine the equivalence of two mean vectors and the Mardia-Watson-Wheeler test to assess for differences in sample distributions. Correlation coefficients and mean values were taken to be significantly different at $p<0.05$.

\section{Results}

Forward swimming in the postmetamorphic Xenopus froglet is accomplished by rhythmic, synchronous kicking of the newly developed hindlimbs that have superseded the now resorbed larval tail as the animal's sole mechanism for body displacement (Combes et al., 2004). Concomitant with this switch in locomotor mode, the laterally positioned eyes of the tadpole migrate dorsofrontally to a position that enables a substantial degree of binocular overlap in the young adult (Grant and Keating, 1986). In a first step, therefore, we assessed how the changes in body plan and propulsive strategy affects ocular motor control by exploring whether potential gaze-stabilizing eye movements might occur during the expression of locomotory behavior.

\section{Limb kinematics and eye movements during swimming}

High-speed video analyses of free-swimming froglets revealed a wide range of displacement velocities in episodes expressed by an individual animal (Fig. 1A1) as well as between different animals (Fig. 1A2). Presumably, this variability reflects an adaptive capability for satisfying different behavioral demands that range from slow food-seeking to rapid escape from predation. Swimming bouts typically consisted of several consecutive propulsive cycles (up to 6 in our experimental conditions; see Materials and Methods) generally with similar peak velocities, although there were some episodes where the velocity progressively increased or decreased during an episode, or varied abruptly from cycle to cycle. Peak velocities ranged from 9.7 to $64 \mathrm{~cm} / \mathrm{s}$ (mean $23.5 \pm 16.6$ $\mathrm{cm} / \mathrm{s} ; n=42$ cycles from 6 animals) with the maximal acceleration observed in our experiments attaining $36.8 \mathrm{~m} / \mathrm{s}^{2}$. Swimming velocity also varied according to the number of cycles expressed within a given episode. Thus, fast swimming froglets typically performed only a few kicks per episode but at high frequency, whereas during slower swimming, they expressed longer episodes with more cycles of longer duration (Fig. 1A).

Each swim cycle started with an extension of the knee joint (Fig. $1 B$, position 1 ) followed by a bilaterally synchronous extension of all hindlimb joints during the main part of the propulsive phase (position 2), which ended when the limbs were fully extended (position 3). Subsequently, a gliding phase occurred, which could be of variable duration, before the hindlimbs returned to their initial position by a flexion of all leg joints in preparation for the next kick cycle (position 4). Despite the considerable variability in the gliding phase interval between limb extension and flexion, peak velocities and cycle durations were strongly negatively correlated, both for a given animal and in pooled measurements from different animals, as indicated by regression analysis of the scatter plots in Figure $1 C$ (single animal: $r^{2}=0.62, p<0.001, n=24$ cycles, blue regression line; group data: $r^{2}=0.46, p<0.001, n=42$ cycles, black line).

To determine whether ocular movements occurred during swimming activity, we next made simultaneous video recordings of the eyes and legs of animals that had their heads held stationary but with the hindlimbs free to perform spontaneous kicking movements. Moreover, in these head-restrained limb-free preparations, the bilateral labyrinthine end organs had been removed and the optic nerves cut (see Materials and Methods). Under such deafferented conditions, therefore, any ocular motion associated with hindlimb kicking was occurring in the absence of normal head displacement and optokinetic influences. As seen in the experiment of Figure $1 D$, which shows a video tracking analysis of the right eye in relation to the right hindlimb position of a semiintact preparation during a representative spontaneous kick, ocular and limb movements were expressed in strict coordination. The principal eye displacements (Fig. 1D1, orange arrow, D2) occurred during each limb extension (Fig. 1D3, images 1-3), which corresponded to the forward propulsive phase observed during unrestrained swimming (Fig. $1 B$, images 1-3) and generally consisted of two overlapping trajectories from the resting position (Fig. 1D1, green line at position 1): inward rotation (up to $\sim 20^{\circ}$ ) of the eye toward the nose (Fig. $1 D 1$, bottom, thick orange line at position 2) at the beginning of the hindlimb extension, which was generally accompanied by an ocular retraction as the extension phase proceeded. Eye retraction was maximal by the time the hindlimb had reached full extension (Fig. 1D1, purple line at 3 in image $3, D 3, B$, image 3 ). The eye then returned toward its original resting position during the subsequent phase of hindlimb flexion (Fig. 1D1, turquoise line at image 4). Finally, the kick-timed motion of one eye occurred simultaneously with oppositely directed positional changes of the contralateral eye (Fig. 1D2,D3), thereby resulting in an overall inward (convergent) movement of both eyes toward each other.

These kinematic data therefore indicated that limb-based locomotion in the young adult frog is indeed accompanied by rhythmic ocular motion, predominantly comprising a convergent rotation and retraction of the two eyes during the powerstroke (extension) phase of each kick cycle. Furthermore, the finding that this functional relationship persisted in the absence of both otolith and visual inputs was the first indication that neural signals other than those arising from the sensing of head and/or retinal image motion must underlie the coupling process. 
A1

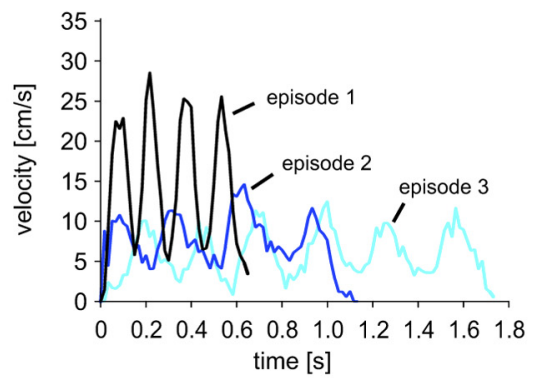

A2

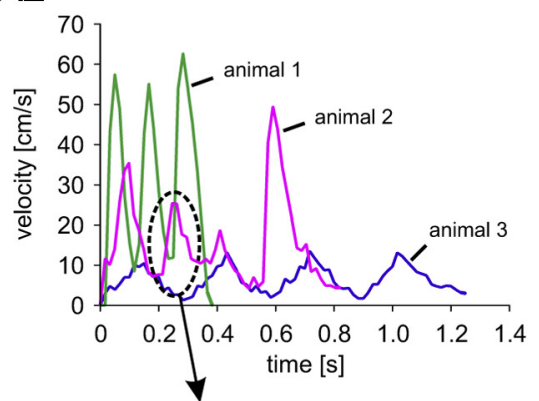

B

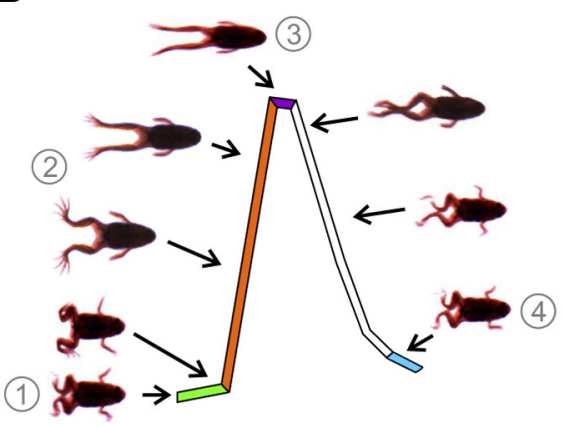

C

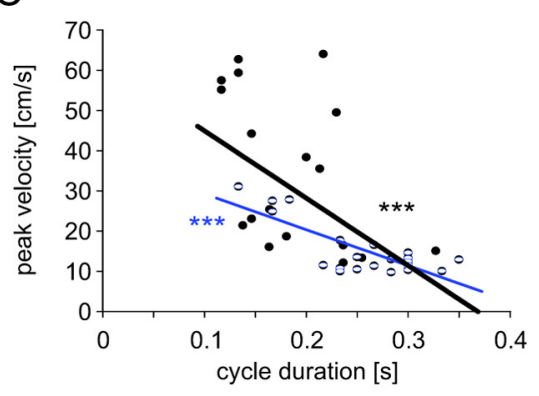

D1
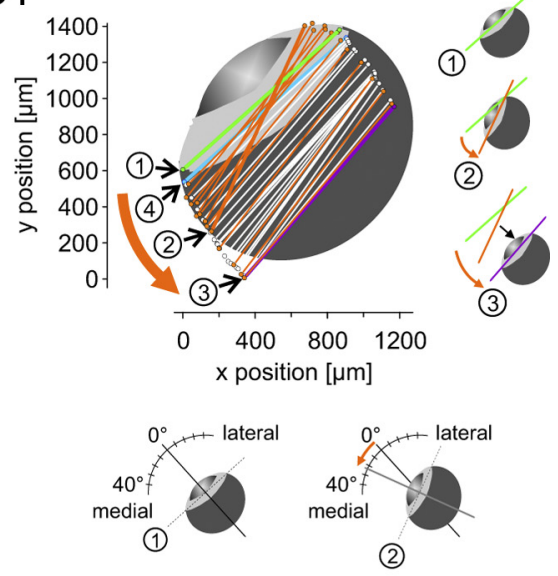

D2

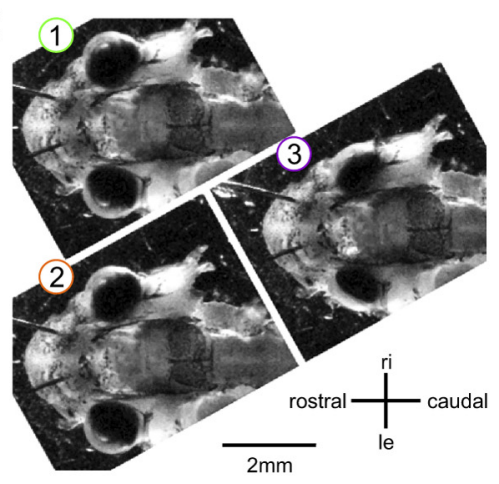

D3
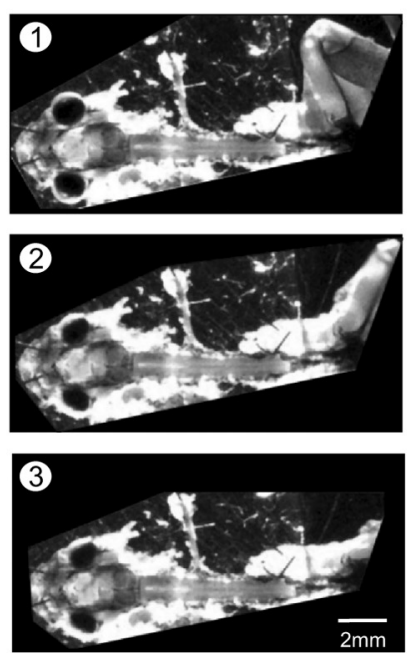

Figure 1. Kinematics of limb and eye movements during swimming activity of young postmetamorphic Xenopus frogs. $\boldsymbol{A}-\boldsymbol{C}$, Analysis of free swimming behavior. A1, Plots of displacement velocity versus time during three different swim episodes from a single animal. Episodes comprised $4-6$ cycles and differed considerably in their peak velocities and durations. A2, Three episodes from different animals, again showing very different peak velocities and episode durations. $\boldsymbol{B}$, Velocity versus time trajectory of a typical single swim cycle (indicated in A2) with corresponding pictograms showing body and limb positions at successive points during the initial propulsive (bilateral hindlimb extension), gliding (limbs held extended), and recovery (bilateral limb flexion) phases. C, Peak velocity versus cycle duration during swim episodes in one animal (blue data points) and pooled measurements from six different animals (black points). In both cases, the coefficients of linear regression were significantly negative (single animal, $r^{2}=0.62$; group, $\left.r^{2}=0.46\right) .{ }^{* *} p<0.001$. D, Ocular movements associated with swimming-related hindlimb kicking. D1, Schematic of the right eye at resting position showing sequential positional changes during a single kick cycle analyzed from a high-speed video recording of a head-restrained froglet with freely moving hindlimbs. Eye motion during limb movement is indicated by changes in the $x$ - $y$ coordinates of two marker spots placed on either side of the eye that were tracked through individual frames of the video sequence and connected by lines for better visualization. The green line (1) indicates initial resting position; orange lines show positional changes of the eye related to hindlimb extension with the thick orange line (2) indicating the maximal inward rotation during limb extension; purple line (3) denotes fully retracted eye position at the end of limb extension; white lines show release from retraction during hindlimb flexion with the turquoise line (4) depicting the eye position at the end

Extraocular motor activity during

fictive swimming in vitro

To further assess the extent to which intrinsic CNS signaling, rather than sensory feedback, underlies this limb-eye movement coordination, we recorded extraocular and spinal motor activity in isolated brainstem/spinal cord preparations (Fig. 2A1) and therefore in the complete absence of all movementrelated sensory feedback. As previously reported (Combes et al., 2004; Beyeler et al., 2008), such in vitro preparations continue to express spontaneous episodes of fictive locomotion in which the spinal CPG circuitry expresses the rhythmic motor burst pattern that would normally drive hindlimb kicking movements in vivo. Thus, as seen in Figure 2A2, ipsilateral antagonistic (flexor/ extensor) limb nerves were active in strict alternation, whereas homologous left-right motor nerves expressed inphase bursting, in a manner appropriate for producing alternating cycles of hindlimb extensions and flexions in bilateral synchrony. In addition, a direct monitor of CPG activity in the lumbar cord region was evident more rostrally at the thoracic cord level (Fig. 2A2, top Th2 traces) where left-right motor bursts occurring in-phase with extensor nerve activity are responsible for coordinating dorsal trunk muscle contractions with limb kicking during actual locomotion (Beyeler et al., 2008). Thus, although generally shorter and slower than swimming episodes produced by the intact animal, the in vitro motor patterns of the isolated brainstem/spinal cord preparation exhibit burst phase relationships that corresponded closely to limb and trunk movements in vivo.

In such isolated preparations, simultaneous recordings from the bilateral motor nerve branches that normally innervate the medial rectus (MR, Fig. 2B1, left) and lateral rectus (LR; Fig. 2B1, right) extraoc-

$\leftarrow$

of the kick cycle. For the purpose of illustration, the two overlapping steps of ocular motion (rotation and retraction) during limb extension are depicted separately in the small pictograms at right. The maximal angle of medial (inward) rotation was $23.5^{\circ}$ from resting position ( $0^{\circ}$; bottom pictograms). The numbered (and colored) eye positions in $\mathbf{D 1}$ correspond to the pictograms and trajectory colorations in $\boldsymbol{B}$ as well as to the numbering of the single video frames of eye positions in $\mathbf{D 2}$. D3, Wider angle images showing eye and associated right hindlimb positions during a leg extension (numbering corresponds to $\boldsymbol{B}, \mathbf{D 1}, \mathbf{D 2}$ ). Positional changes of the right eye were mirrored by those of the left eye to produce coincident, convergent ocular movement in each cycle. 
A1

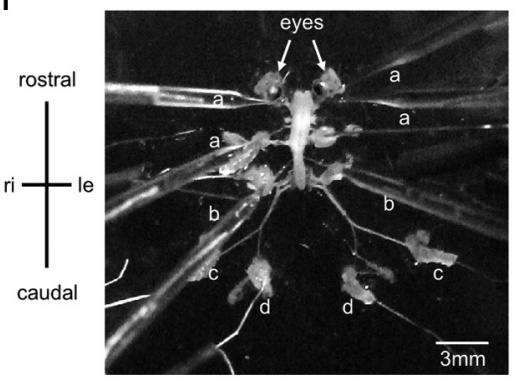

B1

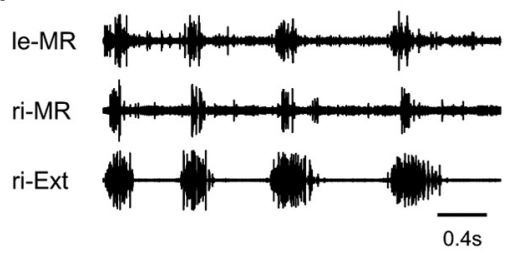

A2

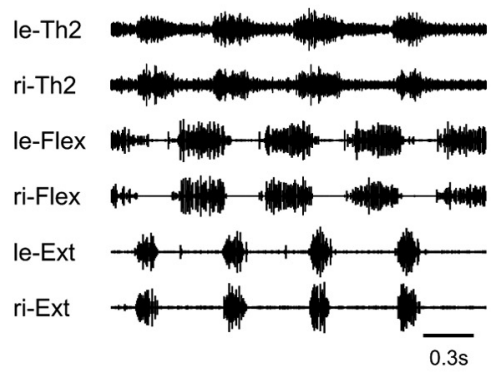

B2
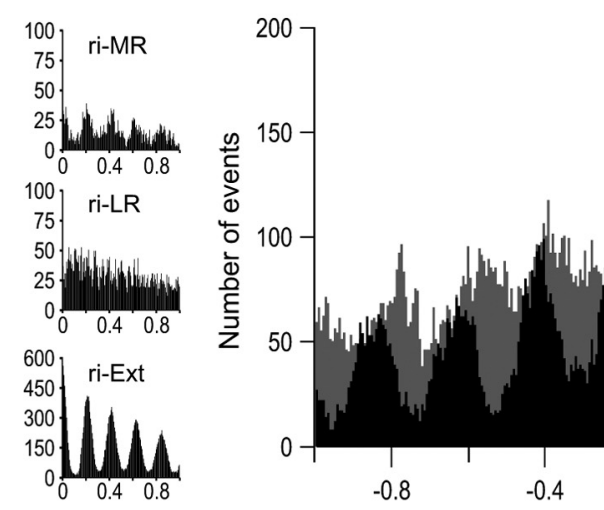

C
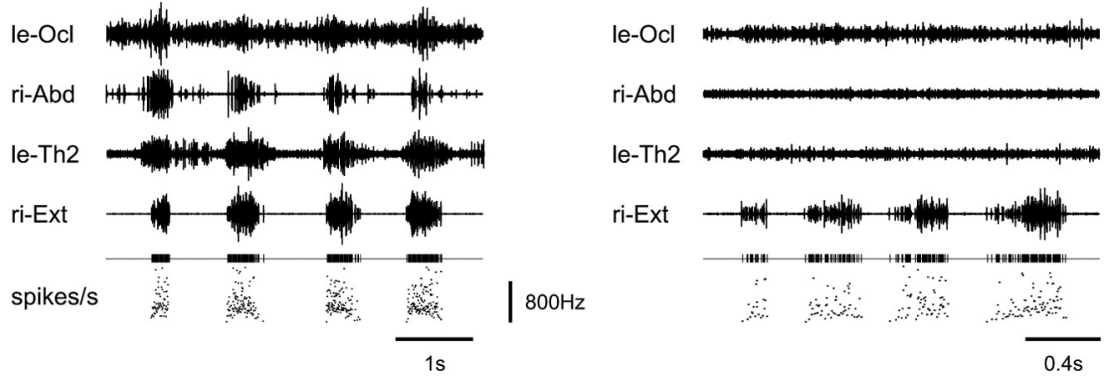

Figure 2. Spinal CPG and extraocular motor activity during spontaneous fictive swimming in vitro. A1, Photograph of the isolated brainstem/spinal cord preparation (ventral side up) showing recording sites from motor nerve branches innervating the left (le) and right (ri) hindlimb tibialis anterior (ankle flexor, Flex, c) and plantaris longus (ankle extensor, Ext, d) muscles, the bilateral ventral roots of the second thoracic (Th2) cord segment (b), and bilateral motor nerves to extraocular muscles (a). $\boldsymbol{A 2}$, During an episode of spontaneous fictive swimming, ipsilateral Ext and Flex limb nerves were active in alternation, whereas homologous bilateral nerves expressed in-phase bursting. Th2 ventral root activity was limb extensor burst-timed. B1, Simultaneous recordings of left and right medial rectus (MR) nerve branches (left) that arise from the cranial oculomotor nerves ( $0 \mathrm{cl})$ and both lateral rectus (LR) nerve branches (right) arising from the cranial abducens nerve (Abd) along with right limb extensor motor nerve during fictive swimming. In both cases, extraocular motor discharge occurred in bilateral coordination with rhythmic extensor nerve bursting. B2, Event correlation analysis of spino-extraocular motor coupling during fictive swimming in a different preparation. Left, Individual auto-correlograms for ri-MR, ri-LR, and ri-Ext nerve discharge. Right, Corresponding crosscorrelograms showing a strong in-phase relationship between the Ext and MR nerve bursts (black plot; $r^{2}=0.56, p<0.001, n=$ 16 cycles). In the same fictive swim episode, LR activity was not significantly coupled to Ext bursts $(p>0.05, n=16)$, despite evidence of Ext-timed LR modulation in the corresponding cross-correlogram (gray plot). C, Bilaterally synchronous, extensor burst-timed activity recorded from the le- $0 \mathrm{Cl}$ and ri-Abd nerves during fictive swimming. Spino-extraocular motor coupling occurred during swim episodes with strong extensor discharge and associated thoracic (Th2) ventral root bursting (left), but not during weaker episodes (right; compare instantaneous frequencies of Ext nerve firing; bottom) when Th2 activation also failed to occur. ular muscles also revealed activity that was coordinated with lumbar motor bursts during episodes of fictive locomotion. Specifically, motoneurons in both extraocular nerves were active in bilateral synchrony and in-phase with hindlimb extensor motoneuron bursting. This raw data evidence for such spino-extraocular coupling was further supported by autocorrelation and cross-correlation analyses, which, respectively, provide quantitative tests of recurrent rhythmicity within, and the extent of coupling between, activity in individual nerve recordings. An example of such analyses, for a different experiment from those of Figure $2 B 1$, is shown in Figure 2B2. Here, ri-MR activity was both strongly rhythmic (see autocorrelogram, top left) and tightly coupled with extensor nerve bursting as evidenced by the clearly defined central peak and satellite distributions in the corresponding cross-correlogram (Fig. 2B2, right, black plot). Throughout the same fictive swim sequence, however, LR nerve activity was tonic rather than rhythmic (see autocorrelogram, middle left), although a certain degree of phasic discharge in time with Ext bursting was evident in the corresponding cross-correlogram (Fig. 2B2, right, gray plot). Together, these and other equivalent data showed that both antagonistic motor nerves responsible for horizontal eye movements in vivo can coactivate with bilaterally synchronous limb extensor bursting during locomotor rhythmogenesis in the isolated CNS, although MR motoneurons consistently display the strongest extensor-timed coupling of the two extraocular motor pools. Presumably, the tonic component of LR discharge that persists throughout hindlimb flexion (when MR firing ceases) assists in returning the eye to its initial resting position before the start of the next locomotor cycle. Thus, the cycle-by-cycle recovery of the eyes to their starting position is achieved by the net effect of a termination in MR nerve burst activity along with a persisting, elevated level of tonic LR nerve discharge during the limb flexion phase.

In principle, the activation of horizontal extraocular motoneurons in time with fictive limb extension could arise in brainstem locomotor command regions and/or the midbrain reticular formation, both of which play a crucial role in initiating and maintaining locomotor behavior (Saitoh et al., 2007; Le Ray et al., 2011). However, several observations argued strongly against such a supraspinal origin. First, rhythmic discharge that might have been 
A1

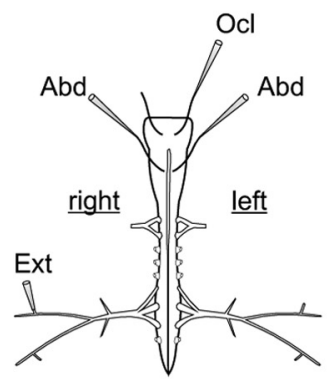

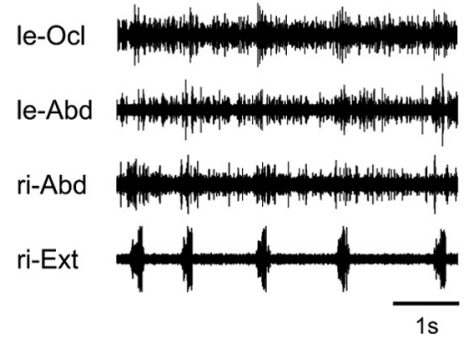

A2

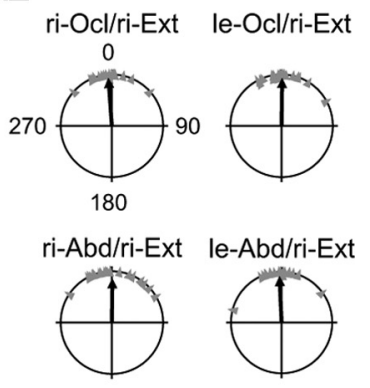

B1

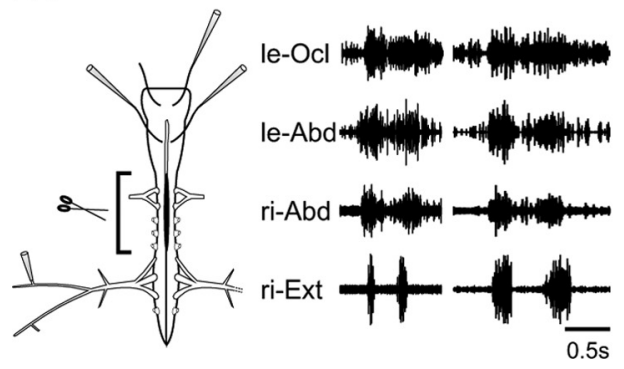

B2

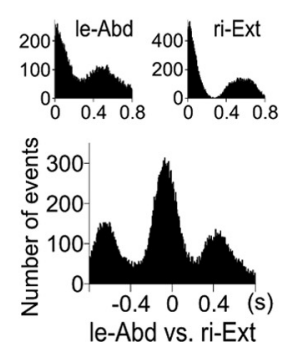

B3
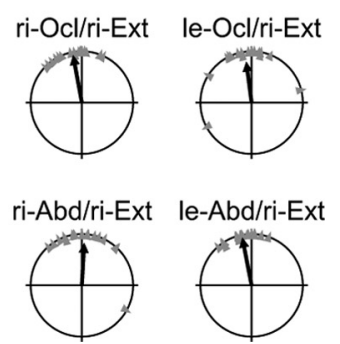

Figure 3. A sagittal midline separation of the cervicothoracic segments does not affect lumbar CPG-extraocular motor coupling. $\boldsymbol{A}$, Control. A1, Schematic of the intact isolated preparation indicating recording protocol (left) and motor activity recorded from the le-OCl, bilateral Abd, and ri-Ext nerves during spontaneous fictive swimming (right). A2, Corresponding circular phase plots of burst onsets in left and right $0 \mathrm{cl}$ (top) and $\mathrm{Abd}$ (bottom) nerves relative to ri-Ext burst onsets showing strongly significant phase synchrony (i.e., near $0^{\circ}$ ) in all cases (ri- $0 \mathrm{cl}$ : mean vector direction $\mu=355.12^{\circ}$, vector length $r=0.94$, phase distribution $Z=15.85$; le- 0 dl: $\mu=0.53^{\circ}, r=0.93, Z=16.58$; ri-Abd: $\mu=0.73^{\circ}, r=0.88, Z=14.59$; le-Abd: $\mu=357.41^{\circ}, r=0.92, Z=15.92$; $p<0.001, n=19$ cycles from 4 animals for all plots). $\boldsymbol{B}$, Postlesion. $\boldsymbol{B} 1$, Schematic of the same preparation after a mid-cord (C1-Th3) separation (left) and samples of recordings (right) from the same motor nerves as in $\boldsymbol{A}$. B2, Auto- (top) and cross-correlations (bottom) for le-Abd versus ri-Ext activity indicating rhythmicity and the maintenance of strong extensor-Abd burst coupling $\left(r^{2}=\right.$ $0.83, p<0.001, n=8$ cycles). $B 3$, Circular analysis (pooled data from 4 animals) showing no postlesion alterations in burst onset synchrony (compare with $A \mathbf{2})\left(\mathrm{ri}-0 \mathrm{cl}\right.$ : $\mu=349.42^{\circ}, r=0.94, Z=$ 15.05; le-0cl: $\mu=352.07^{\circ}, r=0.81, Z=10.45 ;$ ri-Abd: $\mu=2.96^{\circ}, r=0.83, Z=11.7$; le-Abd: $\mu=348.83^{\circ}, r=0.96, Z=15.65 ; p<0.001, n=17$ cycles for all plots).

related to locomotion was never observed in the extraocular motor nerves of isolated CNS preparations after removal of the spinal cord or even after a restricted lesion was made to the lumbar cord to abolish local hindlimb CPG activity $(n=3$; data not shown). Further evidence for a strictly spinal origin of the locomotor signals to extraocular motoneurons was also apparent in recordings of intact brainstem/spinal cord preparations, such as those illustrated in Figure 2C, where spontaneous fluctuations in extensor burst intensities occurred in different bouts of fictive kicking. During typical robust limb motor rhythmicity (Fig. 2C, left), phase-coupled bursting was expressed in the brainstem extraocular motor nuclei as well as in intervening thoracic spinal segments that receive an ascending drive from the limb CPG network (Beyeler et al., 2008). However, spino-extraocular motor coupling disappeared in all preparations $(n=8)$ whenever the strength of ongoing CPG activity decreased spontaneously to an extent where burst activation in the thoracic cord region also failed to occur (Fig. 2C, right). Therefore, together these observations support the conclusion that the neural signals for locomotor-extraocular motor coupling arise in the spinal cord, and specifically, from the lumbar CPG network itself.

\section{Effects of CNS lesions on hindlimb-extraocular motor coordination}

To further explore the spino-extraocular coupling in swimming froglets, a series of specific lesions (see Materials and Methods) were made to in vitro brainstem/spinal cord preparations to locate the anatomical substrate for the transmission of the coordinating signals from the lumbar cord to the brainstem extraocular motor nuclei. In larval Xenopus, spinal efference copies are con- veyed rostrally from axial CPG circuitry by projection pathways that initially traverse the cord near their segment of origin to ascend on the opposite side to the brainstem abducens nuclei (Lambert et al., 2012). Therefore, in a first series of in vitro lesions in young adult preparations, we examined the effects of a longitudinal midline section from the obex through the cervicothoracic spinal region (from cord segments $\mathrm{C} 1$ to $\mathrm{Th} 3$ ), which in the tadpole disrupts the first stage transmission of CPG-driven bursts to the bilateral abducens nuclei and consequently suppresses spino-extraocular coupling. However, in contrast to this lesion's effects in larval in vitro preparations (Lambert et al., 2012), the coupled pattern recorded in the intact control froglet CNS during fictive swimming (Fig. 3A1) appeared to remain unaffected by a cervicothoracic sagittal section (Fig. 3B1). Quantification of the phase relationships and event auto-correlations/cross-correlations between hindlimb extensor and extraocular motor activity during fictive leg kicking ( $n=4$ animals; see Fig. 3 legend for statistics) confirmed the maintained lumbar CPG influence on both MR/ Ocl and LR/Abd nerve activity after this lesion (Fig. 3B2,B3; compare with A2), specifically involving a persistent discharge synchrony between homolateral hindlimb extensor motoneurons and activity in the two extraocular motor nerves (Fig. 3, compare $A 2, B 3$ ). This strongly indicates, therefore, that in young adult Xenopus the principle efference copy-mediating pathways ascend ipsilaterally in the spinal cord, and not contralaterally as in premetamorphic larvae.

We next tested for the presence of a further midline crossover pathway in the brainstem at the level of the abducens nuclei, which was previously found in larval Xenopus to mediate spinal CPG coupling with MR motoneurons in the more rostral oculo- 

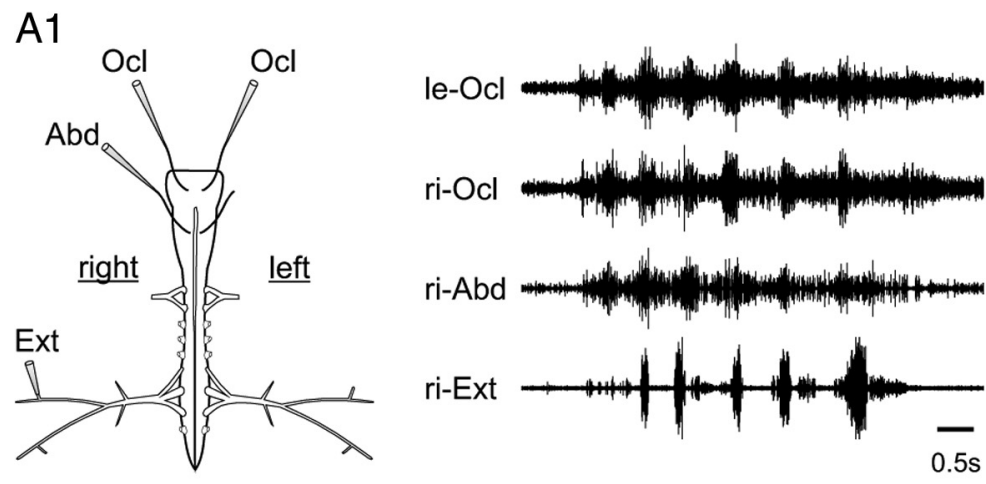

A2

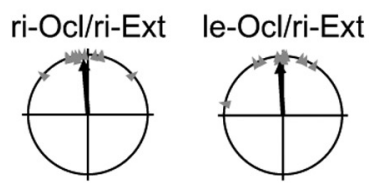

ri-Abd/ri-Ext le-Abd/ri-Ext
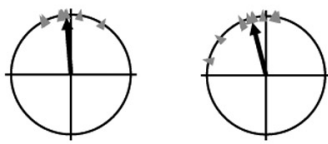

B1
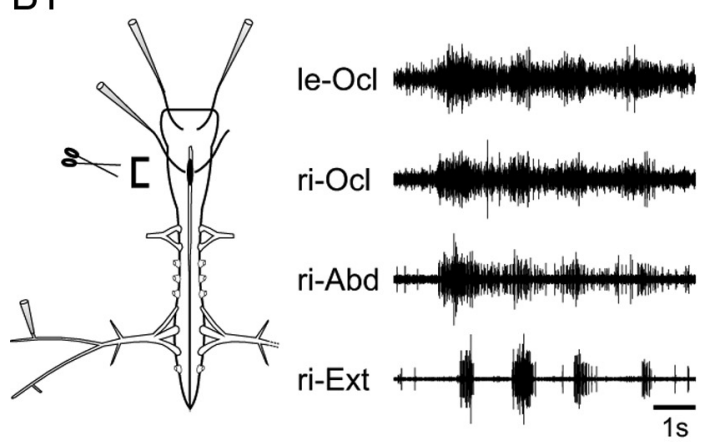

B2
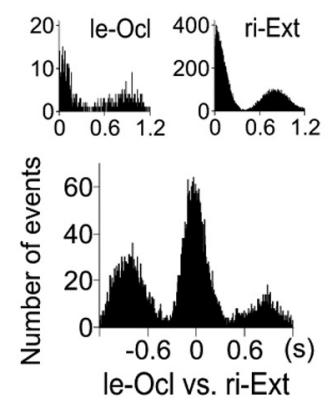

C

D1

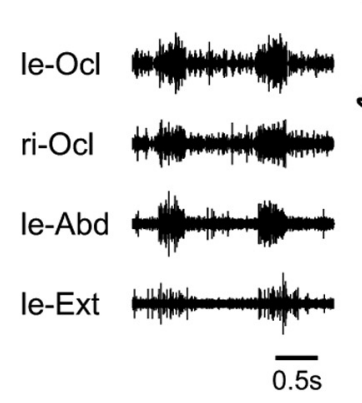

D1

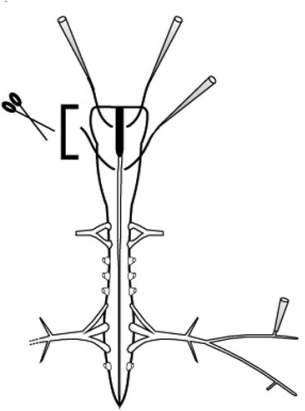

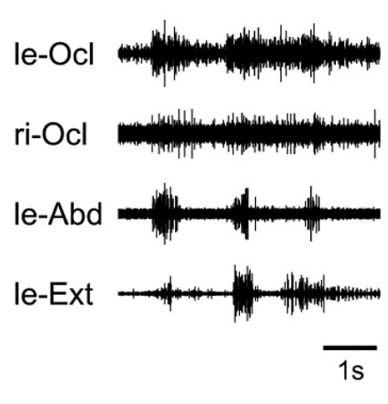

\section{B3}

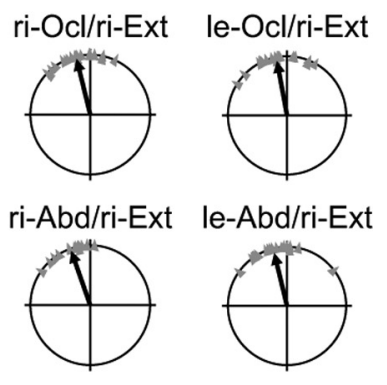

D2

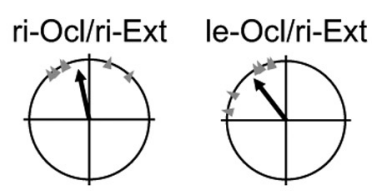

ri-Abd/ri-Ext le-Abd/ri-Ext
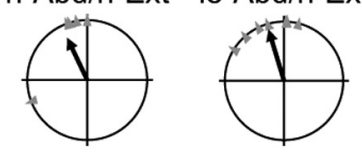

Figure 4. Sagittal midline lesions to the brainstem do not alter lumbar CPG-extraocular motor coupling. A, Control. A1, Schematic of the isolated preparation indicating recording protocol (left) and motor activity recorded from the bilateral $0 \mathrm{Cl}$, ri-Abd, and ri-Ext nerves during spontaneous fictive swimming (right) before a restricted sagittal midline incision at rhombomere 5. A2, Corresponding circular phase plots (data from 4 animals) of burst onsets in left and right $0 \mathrm{Cl}$ (top) and Abd (bottom) nerves relative to ri-Ext burst onsets showing strongly significant phase synchrony in all cases (ri-0cl: $\mu=355.71^{\circ}, r=0.93, Z=10.28$; le- $0 \mathrm{cl}: \mu=356.65^{\circ}, r=0.9, Z=9.61 ;$ ri-Abd: $\mu=354.99^{\circ}, r=0.96, Z=8.24 ;$ le-Abd: $\mu=345.88^{\circ}, r=0.91, Z=9.88 ; p<0.001$, $n=12$ cycles for all plots). $\boldsymbol{B}$, Postlesion. $\boldsymbol{B}$, Schematic of the same preparation after the rhombomere 5 lesion (left) and sample recordings (right) from the same motor nerves as in $\boldsymbol{A}$ during fictive swimming. B2, Event correlation analysis confirming the expression of le- 0 cl and ri-Ext rhythmic bursting that remained strongly coupled $\left(r^{2}=0.74, p<0.001, n=21\right.$ cycles). B3, Circular phase analysis (data from 4 animals) showing the persistence of burst onset synchrony (relative to ri-Ext) after the lesion (compare with $A 2$ ) (ri-0cl: $\mu=346.24^{\circ}, r=0.94, Z=16.92, n=19 ;$; le-0cl: $\mu=$ $349.3^{\circ}, r=0.93, Z=15.51, n=18 ;$ ri-Abd: $\mu=340.85^{\circ}, r=0.96, Z=12.92, n=14 ;$ le-Abd: $\mu=346.53^{\circ}, r=0.92, Z=15.34, n=18 ; p<0.001$ in each case). Coordinated rhythmic bursting in le-Ext, le-Abd, and bilateral Ocl nerves (C) during swimming activity remained unaffected by a complete sagittal separation of the midbrain (D1). D2, Postlesion phase analysis (data from 3 animals) of burst onsets relative to ri-Ext bursts showing maintained burst synchrony (ri- $0 \mathrm{cl}: \mu=348.04^{\circ}, r=0.86, Z=4.46, p<0.001, n=6$ cycles; le- $0 \mathrm{cl}: \mu=322.23^{\circ}, r=0.91, Z=4.92$, $p<0.001, n=6$; ri-Abd: $\mu=33.94^{\circ}, r=0.79, Z=3.08, p<0.05, n=5$; le-Abd: $\mu=343.11^{\circ}, r=0.91, Z=5.85, p<0.001, n=7$ ).

motor nucleus (Lambert et al., 2012). Accordingly, a restricted sagittal midline section was made in the hindbrain of adult preparations at rhombomere 5 (Fig. 4; see Materials and Methods) where the abducens nuclei and their internuclear neurons are located (Straka et al., 2001). In the tadpole, these midline crossing interneurons project to the oculomotor nucleus on the opposite side to excite contralateral MR motoneurons, thereby coupling synergistic MR and LR motoneurons in a manner required for conjugate movements of the two eyes. However, in direct contrast to the disruption of spino-MR motor coupling during fictive swimming in tadpole preparations (Lambert et al., 2012), the timing and magnitude of spinal CPG-driven bursts in both the left and right Ocl nerves of the adult CNS during fictive leg kicking (Fig. 4A1,A2) remained unaffected by this particular lesion (Fig. 4B1-B3): event correlation and circular phase analyses $(n=$ 4 animals) confirmed a similar synchronization of rhythmic hindlimb extensor, MR/Ocl, and LR/Abd motor nerve discharge before and after the lesion (Fig. 4B2,B3). These findings therefore indicated that the efference copy signals underlying spinoextraocular motor coupling do not have major access to their MR motor targets through midline crossing pathways involving neither abducens internuclear neurons, as evident from Figure 4, C and $D$, nor any direct crossover pathways that might exist between the oculomotor nuclei themselves. Although a longitudi- 
A

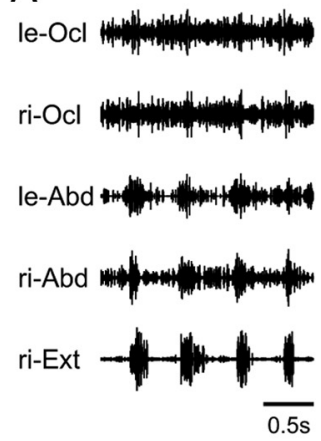

C

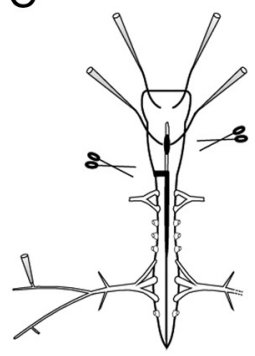

B1
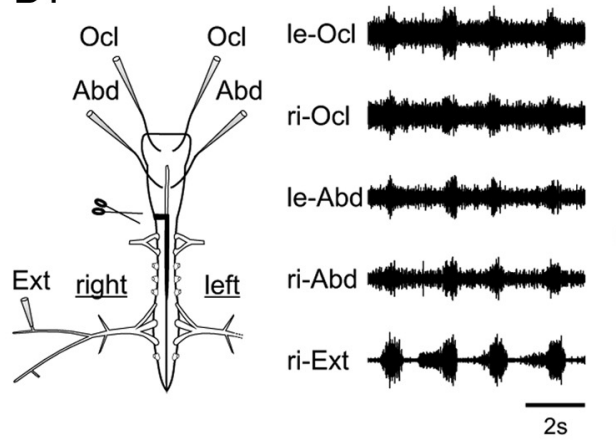

B2

ri-Ocl/ri-Ext le-Ocl/ri-Ext

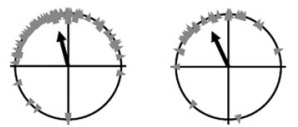

ri-Abd/ri-Ext le-Abd/ri-Ext
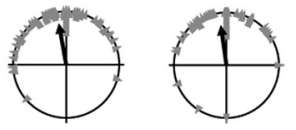

$\mathrm{D}$

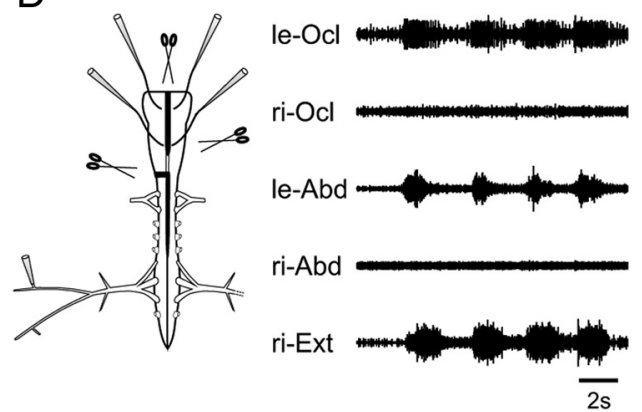

Figure 5. Effects of a rostral cord hemisection in combination with sagittal spinal and brainstem lesions on lumbar CPG-extraocular motor coupling. Swimming-related activity recorded from the indicated nerves of an isolated preparation swimming before $(\boldsymbol{A})$ and after $(\boldsymbol{B} 1)$ a hemisection at the right obex plus a sagittal separation of the cervicothoracic (C1-Th3) cord segments (see schematic). B2, Corresponding circular phase plots of postlesion burst onsets in bilateral Ocl (top) and Abd (bottom) relative to ri-Ext showing a persistence of synchronous bursting after the two lesions (ri-0cl: $\mu=345.25^{\circ}, r=0.67, Z=30.71, n=69 ;$ le-0cl: $\mu=342.62^{\circ}, r=0.76, Z=41.8, n=72 ;$;i-Abd: $\mu=349.62^{\circ}, r=0.78, Z=44.58, n=74 ;$ le-Abd: $\mu=345.33^{\circ}, r=0.83$, $Z=51.93, n=76 ; p<0.001$ in each case). C, A further sagittal lesion at rhombomere 5 of the same preparation (see schematic) completely suppressed spinal-driven re-Abd activity, whereas the le-Abd nerve (on the intact cord side) continued to express ri-Ext timed bursts. $\boldsymbol{D}$, A complete longitudinal separation of the midbrain now suppressed ri-Ext timed activity in ri-Ocl, but lumbar-driven bursting in le-0cl persisted.

nal sagittal section at this more rostral (diencephalon and mesencephalon) level $(n=3)$ generally resulted in a slight decline in the intensity of Ocl discharge compared with intact control, this latter extraocular activity still remained rhythmic and overall synchronously timed to hindlimb extensor motor bursts during fictive leg kicking episodes (Fig. 4C,D1), as confirmed by the circular phase analyses of the respective motor nerve activities plotted in Figure 4D2.

Despite the evidently predominant role of uncrossed ascending pathways in mediating efference copy signaling in adult $\mathrm{Xe}$ nopus, the extent to which midline crossing trajectories might additionally contribute to spino-extraocular coupling was tested by further lesion experiments that used combinatorial transections of axonal pathways at various spinal and brainstem levels. For example, from the findings described above, a sagittal midline separation of the cervicothoracic cord in conjunction with a cord hemisection at the obex on the right side (Fig. 5B1) would be expected to interrupt the normal ipsilateral CPG influence on Abd and Ocl motor activity on the right side during fictive limbkicking (Fig. 5A), without affecting extensor-timed extraocular motor activation on the left side. However, as seen in the recordings of Figure 5B1 (right), extensor-coupled Abd and Ocl bursting persisted bilaterally after the double lesion, although the relative timing shifted slightly and the coupling strength had decreased as illustrated by the corresponding phase analyses in Figure 5B2. This finding therefore suggested that auxiliary midline-crossing connections in the brainstem (i.e., more rostral to the lesion sites of Fig. 5B1) do indeed contribute to the ascending efference copy pathway. In support of this conclusion, more- over, a further midline sagittal section made to the preparation of Figure $5 A, B$ at the level of the abducens nucleus caused a disappearance of CPG-coupled right Abd activity (Fig. $5 C$ ) while extending the midline separation of rhombomere 5 through rhombomere 1-4 and the mesencephalon to now include both the bilateral abducens and oculomotor nuclei, abolished both spinal-driven Abd and Ocl bursts on the right side (Fig. 5D). However, as would be expected, robust synchronous discharge of the (left) extraocular nerves with limb extensor bursting on the left, still intact side essentially remained unaltered.

In a final experiment in this series, the relative dominance of homolateral versus diagonal ascending projections in conveying locomotor CPG efference copies to the respective Abd and Ocl motor pools was confirmed by completely separating the two sides of the CNS in making a midline section that extended from the oculomotor nuclei until the lower thoracic (Th3) cord region (Fig. 6). Despite this extended longitudinal lesion (Fig. 6, compare $A 1, B 1$ ), the characteristic homolateral synchrony between rhythmic extensor bursts and extraocular motor discharge on both sides generally persisted: although the well-defined Abd and Ocl burst activity found in intact control (Fig. 6Al, right), became less distinct after the midline separation (Fig. 6B1, right), a quantification of the spino-extraocular phase relations confirmed the maintenance of largely unaltered coupling (Fig. 6, compare $B 2, A 2)$.

Finally, given the known role of the cerebellum in both locomotor (e.g., Armstrong et al., 1997) and extraocular motor control (e.g., Kheradmand and Zee, 2011), this supraspinal structure might also be involved in mediating the spino-extraocular cou- 
A1

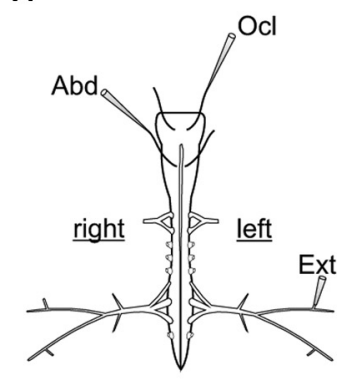

B1

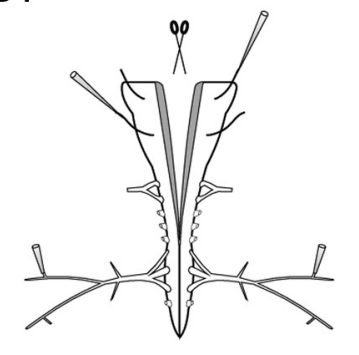

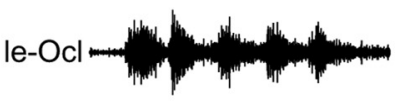
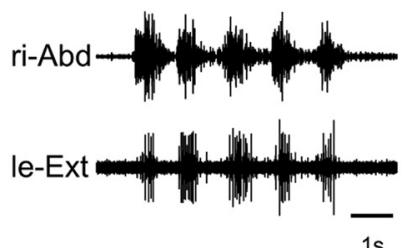

1s

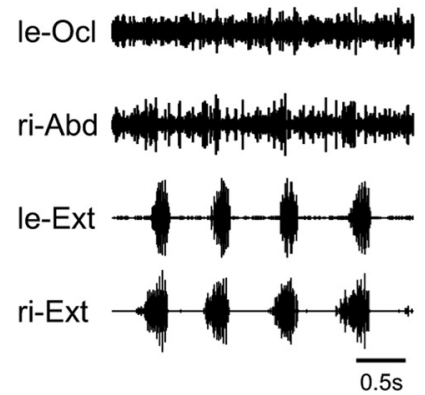

A2

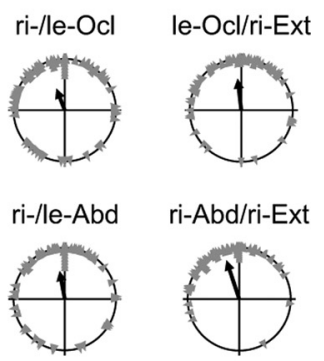

B2

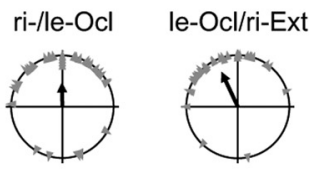

ri-/le-Abd ri-Abd/ri-Ext
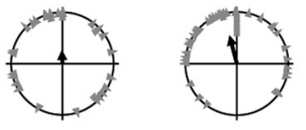

Figure 6. Effects of a longitudinal midline separation of the brainstem and spinal cord on spino-extraocular motor coupling. $\boldsymbol{A}$, Control. $\boldsymbol{A}$, Schematic of recording protocol (left) and motor activity recorded from the le- $0 \mathrm{Cl}$, ri-Abd, and le-Ext nerves during spontaneous fictive swimming (right). $\mathbf{A 2}$, Corresponding circular plots of burst onsets in bilateral $0 \mathrm{cl}$ and $\mathrm{Abd}$ nerves, and $0 \mathrm{cl}$ and Abd onsets relative to ri-Ext bursts, showing phase synchrony in all cases (ri-vs le- $0 \mathrm{cl}$ : $\mu=339.53^{\circ}, r=0.45, Z=16.52, n=82 ; \mathrm{ri}-\mathrm{vs} \mathrm{le}$-Abd: $\mu=349.18^{\circ}, r=0.56, Z=24.35, n=79 ; \mathrm{le}-0 \mathrm{cl}$ vs ri-Ext: $\mu=353.24^{\circ}, r=0.61, Z=29.38, n=80$; ri-Abd vs ri-Ext: $\mu=341.55^{\circ}, r=0.78, Z=49.68, n=82 ; p<0.001$ in all plots). $B 1$, Sample recording of swimming activity in the same preparation after a midline separation from the midbrain until the last thoracic (Th3) segment (see schematic). B2, Postlesion phase analysis still showed a tendency for synchronous activity in rivs le-0cl $\left(\mu=358.44^{\circ}, r=0.48, Z=7.91, p<0.001, n=34\right)$ and ri- vs le-Abd ( $\left.\mu=54.92^{\circ}, r=0.23, Z=2.44, p=0.086, n=32\right)$. Activity in le-0cl $\left(\mu=334.54^{\circ}, r=0.73, Z=18.11\right.$, $p<0.001, n=34)$ and ri-Abd ( $\left.\mu=345.87^{\circ}, r=0.55, Z=15.96, p<0.001, n=52\right)$ also remained in synchrony with ri-Ext bursts.

pling, a possibility that is supported by the presence of CPGtimed activity in the spino-cerebellar pathways of cats during rhythmic motor behavior (Arshavsky et al., 1978a,b,1984,). To test this possibility in Xenopus froglets, therefore, we compared the spino-extraocular motor coupling during fictive limbkicking before and after surgical removal of the cerebellum in isolated preparations $(n=2)$ in which the spinal cord and brainstem otherwise remained intact. However, after this ablation, both the magnitude and the phase relations between Ocl/Abd and Ext nerve activity remained unaltered (data not shown), thus indicating that the cerebellum does not after all intervene in conveying efference copy signals from the spinal cord to the extraocular motor nuclei.

On the basis of the various lesions described above, therefore, and in direct contrast to the dual midline-traversing projections from the spinal CPG to the LR and MR motor pools in larval frogs (Lambert et al., 2012), the anatomical substrate for the corresponding efference copy drive to extraocular motor targets in the young adult consists mainly of homolaterally ascending spinal/ brainstem pathways, with only a minimal contribution by diagonal axonal trajectories. These pathways for spino-extraocular coupling during the propulsive (extensor) phase of adult limbkicking are summarized schematically in Figure 7.

\section{Discussion}

Rectilinear locomotor propulsion in young adult Xenopus frogs is accompanied by nonconjugate eye movements. During each kick stroke, generated by synchronized bilateral hindlimb extensions, both eyes converge, consistent with the requirement to maintain stable binocular vision. During fictive limb kicking in isolated CNS preparations, bilateral MR extraocular motoneurons are synchronously active, in accordance with their production of effective convergent eye movements in vivo. The strict phase coordination between hindlimb extensor and extraocular motoneuron burst activity and the underlying direct spinoextraocular connectivity thus suggests a major role for an intrinsic CPG efference copy mechanism in retinal image stabilization during Xenopus limb-based locomotion.

\section{Coadaptation of gaze-stabilizing eye movements and locomotor strategy}

Minimizing retinal image slip during locomotion is essential to maintain visual acuity (Angelaki and Hess, 2005; Angelaki and Cullen, 2008). For optimal performance, however, it is necessary that the spatiotemporal profile of gaze-stabilizing eye movements matches the organization and dynamics of the particular locomotory modality used. Although locomotion of terrestrial tetrapods with flexible necks generally requires relatively complex $3 \mathrm{D}$ eye movements to stabilize retinal images, the undulatory tail-based swimming of most aquatic vertebrates, such as larval Xenopus (Hoff and Wassersug, 1986; Azizi et al., 2007), necessitates only relatively simple paired (conjugate) ocular movements to counteract head rotational movements that are mainly in the horizontal plane. Indeed, such an eye motion profile has been recently observed during swimming in Xenopus tadpoles (Lambert et al., 2009, 2012), consistent with alternate burst discharge elicited in bilateral synergistic pairs of MR and LR motor nerves (Lambert et al., 2008) during passively induced, semicircular canal-derived horizontal angular vestibulo-ocular reflexes (aVOR) (Straka and Dieringer, 2004; Angelaki and Cullen, 2008).

Although the spatiotemporal needs for gaze stabilization during undulatory larval frog swimming are relatively easy to 


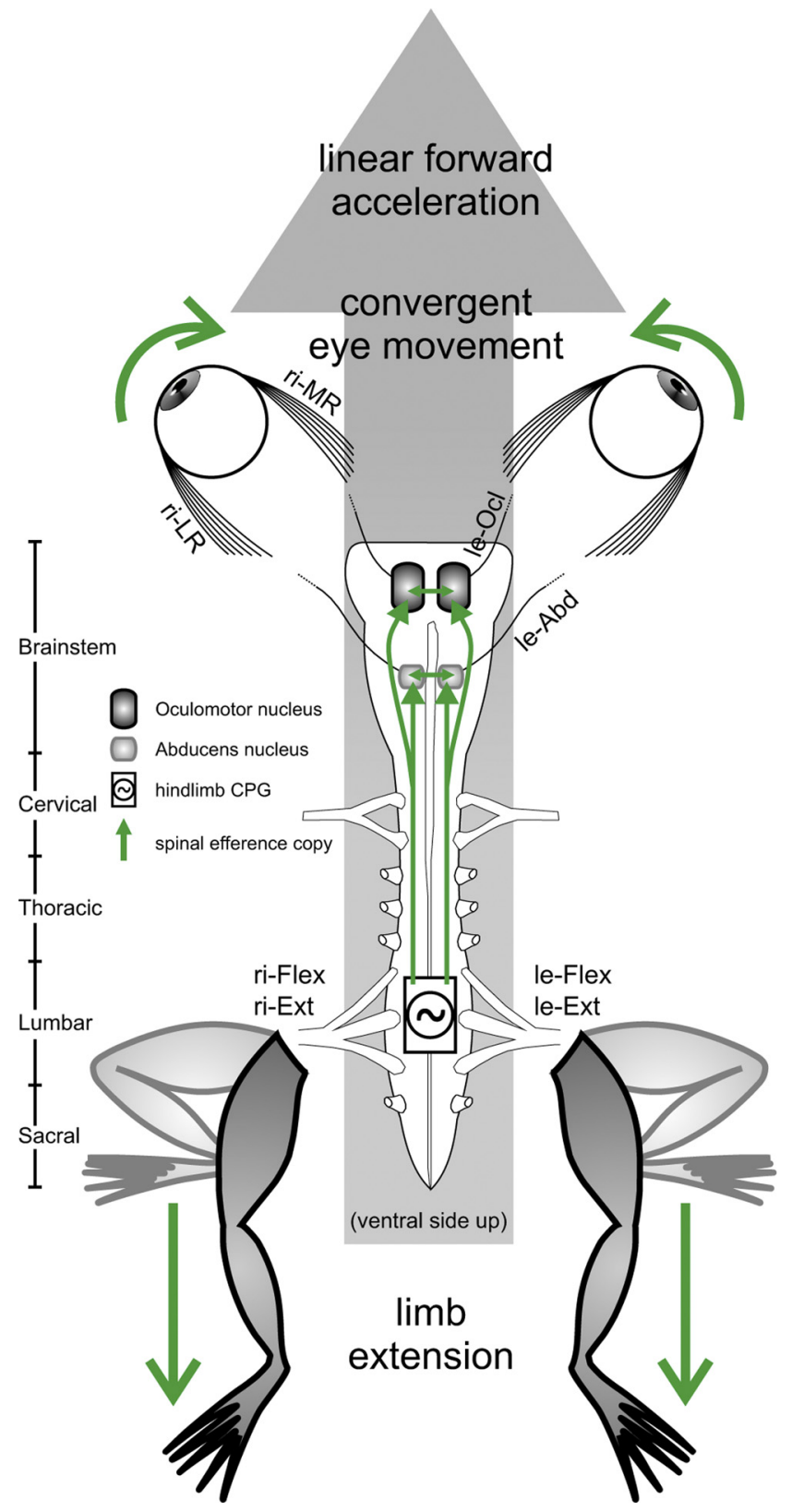

Figure 7. Schematic representation of an intrinsic, nonsensory mechanism for gaze stabilization during rhythmic swimming movements in young adult $X$. laevis. During linear forward acceleration in each cycle generated by bilaterally synchronous hindlimb extensions, a spinal efference copy from the lumbar CPG network is conveyed to the brainstem extraocular motor nuclei via homolaterally ascending pathways to drive phase-coupled contractions of the left and right horizontal extraocular muscles. This in turn produces overall convergent eye movements to offset the disruptive visual consequences of propulsive body/head motion.

predict, it is more difficult to estimate these visual requirements during adult limb-based locomotion. Here, rhythmic swimming movement consists principally of linear forward accelerations and decelerations during the power-stroke (extension) and recovery (flexion) phases, respectively, of the locomotor cycle. The alternating horizontal linear head acceleration/deceleration components, which can be experimentally reproduced by passive translational body movements (Rohregger and Dieringer, 2002), trigger convergent eye movements during each forward phase of the imposed motion (Straka and Dieringer, 2004). These vergent eye movements, which are attributed to linear VOR (IVOR) activa- tion by a particular sector of direction-specific utricular hair cells, represent a conserved vertebrate mechanism as indicated by the occurrence also of similar eye motion patterns in rats during horizontal linear translation (Hess and Dieringer, 1990, 1991).

The eye motion profile observed during passive longitudinal body motion (Straka and Dieringer, 2004) suggested that disconjugate eye movements might also occur during self-generated propulsion. That this is indeed the case was revealed here by monitoring ocular motion in froglets during the spontaneous expression of locomotor-related behavior (Fig. 1). Moreover, the fact that kick-coordinated eye movements occurred in experimental preparations with bilateral labyrinthectomy and optic nerve transection was the first indication (also see below) that nonsensory neural signals were underlying the coupling process. The production of convergent eye movements during each hindlimb extension presumably satisfies the need for focal plane stabilization when the animal approaches a visual target. Although this requirement is self-evident for frontal-eyed, foveate vertebrates with clear binocular fields, such as primates (Angelaki and Hess, 2005), it would seem less relevant to afoveate frogs with their more laterally positioned eyes and visual field orientations, albeit that binocular overlap does occur (Grant and Keating, 1986). Moreover, a substantial proportion of small-field $\left(\sim 5^{\circ}\right)$ binocular neurons have been encountered in the optic tectum of adult frogs (Finch and Collett, 1983; Gaillard, 1985), implying that corresponding rhythmic shifts in the focal plane of both eyes by alternating convergent and divergent movements, and despite the absence of a fovea, are needed to ensure the continuous activation of these neurons and their binocular field representations during self-motion.

Thus, the fundamentally different patterns of compensatory ocular motor responses during larval and adult frog locomotion indicate that the overall characteristics of eye movements that aim toward minimizing retinal image slip are adapted to the spatiotemporal specificities of their distinct propulsive strategies. This in turn highlights the developmental plasticity required to maintain optimal functionality despite the alterations in biomechanical and ecophysiological conditions occurring during metamorphosis. Importantly, moreover, the eye movement profiles occurring during active undulatory tail- and rectilinear limbbased propulsion match those of the respective spatiotemporal properties of the aVOR and lVOR during passive body motion (Straka and Dieringer, 2004), compatible with the conventional view that such vestibular reflexes are the major mechanism for retinal image stabilization during self-movement.

\section{Contribution of efference copy signaling to retinal image stabilization}

Although gaze-stabilizing motor output is classically considered to originate from visuo-vestibular afferent signals (Straka and Dieringer, 2004; Angelaki and Cullen, 2008), recent studies have demonstrated that spinal locomotor efference copies directly drive conjugate eye movements during swimming in Xenopus tadpoles (Combes et al., 2008; Lambert et al., 2012). Furthermore, the CPG-driven extraocular motor activation is alone sufficient to offset visual disturbances without requiring additional sensory feedback because during episodes of stable swimming, the horizontal aVOR is actively suppressed (Lambert et al., 2012). However, the relatively simple spatiotemporal relationship between the tadpole's spinal locomotor command, effective body/ head motion, and resultant visual perturbation might preclude a more general deployment of efference copy signaling in gaze sta- 
bilization during more complex vertebrate locomotor actions, such as limb-based propulsion.

Here, a potentially general role for intrinsic efference copies in gaze control during tetrapod locomotion (Chagnaud et al., 2012) was further confirmed in CNS preparations of adult Xenopus in which fictive hindlimb kicking is expressed spontaneously (Combes et al., 2004; Beyeler et al., 2008). Under such in vitro conditions in the complete absence of any movement-encoding sensory inputs, the functional consequences of a phase-specific coupling predominantly between bilateral MR and hindlimb extensor motoneuron bursting (Fig. 2) matched those of reflexdriven responses to comparable, passively imposed body motion (Wadan and Dieringer, 1994), thereby providing the neural correlate of the convergent eye movements observed during actual limb kicking in vivo. This strongly suggests that locomotor efference copies also contribute to offsetting visual perturbations during limb-based locomotion where considerable linear acceleration/deceleration components are produced during each movement cycle. However, whether the utricular-derived IVOR along with optic flow-elicited visuo-motor reflexes during the forward head/body motion are supplemented or, as for the horizontal aVOR in tadpoles (Lambert et al., 2012), are superseded by the intrinsic feedforward signal to the extraocular motor system remains to be determined. Moreover, although the phasic, albeit weaker, coactivation of LR motoneurons with their ipsilateral MR partners during hindlimb extensor activity appears contradictory to binocular convergence, it is likely that the simultaneous contractions of antagonistic extraocular muscles serve to stabilize ocular position and orientation during cyclic inward displacement.

\section{Adaptive developmental plasticity of efference copy signaling pathways}

In larval Xenopus, the intrinsic efference copy signals are conveyed by a direct, diagonally projecting ascending pathway from CPG circuitry in the rostral cord to contralateral LR motoneurons in the hindbrain abducens nuclei and then to synergistic MR motoneurons on the opposite side of the midbrain via abducens internuclear neurons (Lambert et al., 2012). This double midline-crossing trajectory is thus appropriate for producing conjugate left-right rotational movements of the eyes during tail oscillations. In contrast, our lesion experiments indicated that the spino-extraocular pathway in postmetamorphic juveniles is substantially different, involving homolaterally ascending axons from the lumbar spinal cord to MR (and LR) motoneurons, without a significant involvement of midline-crossing trajectories via abducens internuclear neurons (Figs. 3, 4, 5, and 6). Interestingly, ipsilateral ascending projections from the lumbar CPG have been found to coordinate postural dorsal (thoracic) muscle contractions in synchrony with hindlimb extensor motoneuron bursting during swimming in Xenopus froglets (Beyeler et al., 2008). Moreover, here we have found that an effective efference copy drive to extraocular motoneurons occurs uniquely when coincident thoracic motor burst activation occurs (Fig. 2). It is therefore plausible that the bilateral lumbothoracic coupling pathways also continue rostrally until the brainstem oculomotor nuclei.

Thus, the functional adaptation in spinal CPG-extraocular motor coordination that accompanies the metamorphic transition from larval tail-based to limb-based adult locomotion is paralleled by fundamental changes in the underlying coupling pathways, with the latter's specific trajectories matching the different requirements for minimizing visual field perturbations in the two propulsive locomotor modes. The intrinsic nature of the efference copy mechanism suggests the presence of an inherent reference frame that accounts for the changing behavioral needs during the process of adaptive plasticity and rewiring of the ascending coupling pathways during metamorphosis. Presumably, the switch in spino-extraocular coupling occurs in close association with the emergence of the lumbar CPG network controlling the newly developing hindlimbs and the disappearance of primary axial circuitry with tail resorption (Combes et al., 2004). Especially intriguing in this context is that, at intermediate metamorphic stages, both larval and adult motor systems coexist and are cofunctional, thereby providing the unique opportunity to explore the changing interactions between locomotor and gaze control circuitry as one propulsive strategy emerges and supplants another within the same organism.

\section{References}

Angelaki DE, Cullen KE (2008) Vestibular system: the many facets of a multimodal sense. Annu Rev Neurosci 31:125-150. CrossRef Medline

Angelaki DE, Hess BJ (2005) Self-motion-induced eye movements: effects on visual acuity and navigation. Nat Rev Neurosci 6:966-976. CrossRef Medline

Armstrong DM, Apps R, Marple-Horvat DE (1997) Aspects of cerebellar function in relation to locomotor movements. Prog Brain Res 114:401421. CrossRef Medline

Arshavsky YI, Gelfand IM, Orlovsky GN, Pavlova GA (1978a) Messages conveyed by spinocerebellar pathways during scratching in the cat: I. Activity of neurons of the lateral reticular nucleus. Brain Res 151:479 491. CrossRef Medline

Arshavsky YI, Gelfand IM, Orlovsky GN, Pavlova GA (1978b) Messages conveyed by spinocerebellar pathways during scratching in the cat: I. Activity of neurons of the ventral spinocerebellar tract. Brain Res 151: 493-506. CrossRef Medline

Arshavsky YuI, Gelfand IM, Orlovsky GN, Pavlova GA, Popova LB (1984) Origin of signals conveyed by the ventral spino-cerebellar tract and spinoreticulo-cerebellar pathway. Exp Brain Res 54:426-431. CrossRef Medline

Azizi E, Landberg T, Wassersug RJ (2007) Vertebral function during tadpole locomotion. Zoology 110:290-297. CrossRef Medline

Beyeler A, Métais C, Combes D, Simmers J, Le Ray D (2008) Metamorphosis-induced changes in the coupling of spinal thoracolumbar motor outputs during swimming in Xenopus laevis. J Neurophysiol 100:1372-1383. CrossRef Medline

Chagnaud BP, Simmers J, Straka H (2012) Predictability of visual perturbation during locomotion: implications for corrective efference copy signaling. Biol Cybern 106:669-679. CrossRef

Combes D, Merrywest SD, Simmers J, Sillar KT (2004) Developmental segregation of spinal networks driving axial- and hindlimb-based locomotion in metamorphosing Xenopus laevis. J Physiol 559:17-24. CrossRef Medline

Combes D, Le Ray D, Lambert F, Simmers J, Straka H (2008) An intrinsic feed-forward mechanism for vertebrate gaze stabilization. Curr Biol 18: R241-R243. CrossRef Medline

d'Avella A, Bizzi E (2005) Shared and specific muscle synergies in natural motor behaviors. Proc Natl Acad Sci U S A 102:3076-3081. CrossRef Medline

d'Avella A, Saltiel P, Bizzi E (2003) Combinations of muscle synergies in the construction of a natural motor behavior. Nat Neurosci 6:300-308. CrossRef Medline

Finch DJ, Collett TS (1983) Small-field, binocular neurons in the superficial layers of the frog optic tectum. Proc R Soc Lond B Biol Sci 217:491-497. CrossRef Medline

Gaillard F (1985) Binocularly driven neurons in the rostral part of the frog optic tectum. J Comp Physiol A Neuroethol Sens Neural Behav Physiol 157:47-55. CrossRef Medline

Grant S, Keating MJ (1986) Ocular migration and the metamorphic and postmetamorphic maturation of the retinotectal system in Xenopus laevis: an autoradiographic and morphometric study. J Embryol Exp Morphol 92:43-69. Medline

Hess BJ, Dieringer N (1990) Spatial organization of the maculo-ocular re- 
flex of the rat: responses during off-vertical axis rotation. Eur J Neurosci 2:909-919. CrossRef Medline

Hess BJM, Dieringer N (1991) Spatial organization of linear vestibuloocular reflexes of the rat: responses during horizontal and vertical linear acceleration. J Neurophysiol 6:1805-1818. Medline

Hoff KvS, Wassersug RJ (1986) The kinematics of swimming in larvae of the clawed frog, Xenopus laevis. J Exp Biol 122:1-12.

Kheradmand A, Zee DS (2011) Cerebellum and ocular motor control. Front Neurol 2:53. CrossRef Medline

Lambert FM, Beck JC, Baker R, Straka H (2008) Semicircular canal size determines the developmental onset of angular vestibuloocular reflexes in larval Xenopus. J Neurosci 28:8086-8095. CrossRef Medline

Lambert FM, Beraneck M, Arama J, Homa A, Vidal PP, Eskiizmirliler S, Straka H (2009) Differential swimming dynamics during Xenopus ontogeny: implications for gaze stabilization. Soc Neurosci Abstr 35:813.13.

Lambert FM, Combes D, Simmers J, Straka H (2012) Gaze stabilization by efference copy signaling without sensory feedback during vertebrate locomotion. Curr Biol 22:1649-1658. CrossRef Medline

Le Ray D, Juvin L, Ryczko D, Dubuc R (2011) Supraspinal control of locomotion: the mesencephalic locomotor region. Prog Brain Res 188:51-70. CrossRef Medline

Nieuwkoop PD, Faber J (1956) Normal table of Xenopus laevis (Daudin). In:
A systematical and chronological survey of the development from the fertilized egg till the end of metamorphosis. Amsterdam: North Holland.

Roberts A, Soffe SR, Wolf ES, Yoshida M, Zhao FY (1998) Central circuits controlling locomotion in young frog tadpoles. Ann N Y Acad Sci 860: 19-34. CrossRef Medline

Rohregger M, Dieringer N (2002) Principles of linear and angular vestibuloocular reflex organization in the frog. J Neurophysiol 87:385-398. Medline

Saitoh K, Ménard A, Grillner S (2007) Tectal control of locomotion, steering, and eye movements in lamprey. J Neurophysiol 97:3093-3108. CrossRef Medline

Straka H, Dieringer N (1991) Internuclear neurons in the ocular motor system of frogs. J Comp Neurol 312:537-548. CrossRef Medline

Straka H, Dieringer N (2004) Basic organization principles of the VOR: lessons from frogs. Prog Neurobiol 73:259-309. CrossRef Medline

Straka H, Baker R, Gilland E (2001) Rhombomeric organization of vestibular pathways in larval frogs. J Comp Neurol 437:42-55. CrossRef Medline

Straka H, Baker R, Gilland E (2006) Preservation of segmental hindbrain organization in adult frogs. J Comp Neurol 494:228-245. CrossRef Medline

Wadan K, Dieringer N (1994) Abducens nerve responses of the frog during horizontal linear acceleration: data and model. Biol Cybern 70: 533-540. CrossRef Medline 